\title{
Zuogui Pill on Heat Shock Protein 70 and Ultrastructure of Brain Neurons in Senile Dementia Model Rats
}

\author{
QIANQIAN LI ${ }^{1}$, XIAOLI LI², WEI TIAN ${ }^{1}$, YUFEI CHEN, XI WANG AND XIN WANG ${ }^{1 *}$
}

Beijing University of Traditional Chinese Medicine, The Third Affiliated Hospital of Beijing University of Traditional Chinese Medicine, Chaoyang, Beijing 100029, ${ }^{1}$ Department of Acupuncture and Moxibustion, Capital Medical University Beijing Hospital of Traditional Chinese Medicine, Dongcheng, Beijing 100009, ${ }^{2}$ Department of Encephalopathy, The Third Affiliated Hospital of Beijing University of Traditional Chinese Medicine, Chaoyang, Beijing 100029, China

\section{Li et al.: Zuogui Pill on Heat Shock Protein 70 and Ultrastructure of Brain Neurons}

\begin{abstract}
Alzheimer's disease is a pathological mechanism of chronic degenerative disease of brain function, which has a profound influence on various brain diseases, with the highest incidence in the elderly population. With the deepening of population aging, the incidence of Alzheimer's disease is also on the rise, seriously affecting the quality of life of the elderly population. Therefore, the study of neuronal heat shock protein 70 and its ultrastructure is helpful to enhance the therapeutic effect and improve the quality of life of patients. To observe the effect of Zuogui pill on heat shock protein and ultrastructure of brain neurons in rats with Alzheimer's disease. The experimental rats were divided into model group, anti-cerebral failure control group, Haboin control group, Zuogui pill low-dose group, Zuogui pill high-dose group and sham operation group. The model group was injected with D-galactose intraperitoneal injection and amyloid-beta injection into the hippocampus to prepare an Alzheimer's disease animal model. The two control groups were injected with anti-Nao Shuai capsule and Haberman capsule while modeling. The sham operation group was injected with the same amount of normal saline into the hippocampus of both rats. After successful modeling, acetylcholinesterase of rat brain homogenate was detected by biochemical method, expression of heat shock protein 70 in hippocampus of rats in each group was measured by reverse transcription-polymerase chain reaction and ultrastructural changes of rat brain neurons were observed by transmission electron microscope. Zuogui pill could inhibit acetylcholinesterase activity, reduce apoptosis and stabilize the number of neurons. Ultrastructural observation also showed that hippocampal neurons in each treatment group showed morphological characteristics different from the early apoptosis in the model group. Zuogui pill has a positive effect on increasing the number of neurons surviving and inhibiting cell death. It can effectively promote the repair of nerve cells in the brain, thus improving memory impairment in Alzheimer's disease patients.
\end{abstract}

Key words: Alzheimer's disease, heat shock protein 70, transmission electron microscope, brain neurons

Alzheimer's Disease (AD) is a neurological disorder characterized by cognitive impairment and memory loss. The most common diseases occur in the elderly population and the clinical symptoms include neuron loss, Senile Plaques (SPs) and neuronal fiber tangles. The pathogenesis of senile dementia is very complex and there is a lack of authoritative treatment and prevention measures at present.

In recent years, the incidence of senile dementia has shown an obvious trend of increase. In addition, under the influence of objective factors such as aging population and accelerating pace of urban life, the quality of life of patients with senile dementia has

*Address for correspondence E-mail: wangxinflare@163.com January-February 2022 been confronted with unprecedented challenges ${ }^{[1,2]}$. At present, there is still a lack of authoritative identification of the pathogenesis of senile dementia and the currently recognized pathogenesis mainly includes: Inflammatory mechanism, a neurotoxicity hypothesis, free radical damage theory and oxidative stress theory, etc., ${ }^{[3]}$. Traditional Chinese Medicine (TCM) believes that the loss of medullary sea and insufficiency of renal

\footnotetext{
This is an open access article distributed under the terms of the Creative Commons Attribution-NonCommercial-ShareAlike 3.0 License, which allows others to remix, tweak, and build upon the work non-commercially, as long as the author is credited and the new creations are licensed under the identical terms
}

Accepted 31 January 2022 Revised 04 August 2021 Received 12 January 2021 Indian J Pharm Sci 2022;84(1):121-129 
essence are the main pathogenesis of senile dementia ${ }^{[4]}$. It is certain that the pathogenesis of senile dementia is closely related to the generation of $\mathrm{SPs}^{[5]}$. Age spots are not solubility of plaques in the brain organization, its main composition is extracellular beta-amyloid, autopsy study found that patients with $\mathrm{AD}$ in $\mathrm{AD}$ patients in the body of Amyloid-beta $(A \beta)_{1-42}$ may also appear in the normal brain tissue, the protein in brain tissue of precipitation accumulated a large number of local space and converge into age spots, such change is brain tissue to combat $\mathrm{AD}$ cause related injury of a kind of brain tissue damage metabolic mechanism ${ }^{[6,7]}$. $\mathrm{A} \beta$, there are a variety of physiological functions, the main form ion channels, regulating cholesterol, play is a function of resisting oxidative stress, after precipitation, sedimentary strong antiviral effect, excessive accumulate in brain tissue, can induce a series of inflammatory response and eventually leads to the deterioration of neurons, induce $\mathrm{AD}^{[8]}$.

This study used left to pill as the main prescription drugs for the treatment of senile dementia, research established $\mathrm{AD}$ compound model of rats, observe the left to pill behavior at the level of model rats bone metabolism, oxygen free radical, left to pill on simulation of $\mathrm{AD}$ model rats neurons apoptosis regulation and the influence of ultrastructure, explore out pill action mechanism of prevention and treatment of $\mathrm{AD}$, is left to provide experimental basis for treating senile dementia.

\section{MATERIALS AND METHODS}

\section{AD:}

$\mathrm{AD}$ is a degenerative disease of the central nervous system characterized by memory loss and advanced cognitive decline ${ }^{[9]}$. The main clinical manifestations are the decline of cognitive ability, thinking ability, learning ability and judgment ability. Once AD is diagnosed, patients tend to suffer from decreased mobility and general decline in self-care ability, which not only harms the patients themselves, but also interferes with the quality of life of the whole family ${ }^{[10]}$. As a degenerative disease of the central nervous system, AD mostly occurs in the elderly. In addition to the common diseases mentioned above, there may even be complications of mental disorders, bringing extremely heavy burden to patients and their families.

AD was first named in the early last century, people are more used to call it the senile dementia at home, for the patients with $\mathrm{AD}$, typical pathological changes in the brain for the appearance of age spots, the loss of neurons and nerve fibers formation of entanglement, the emergence of the pathological characteristics will also accelerate the loss of neurons, impairs the development of brain function. The latest survey data shows that the number of $\mathrm{AD}$ patients is on the rise year by year and has been listed as the three most fatal causes of death in critical human health together with tumors and cardiovascular and cerebrovascular disease ${ }^{[11]}$. It is estimated that by 2050 , there will be 150 million AD patients in the world. Therefore, the current focus and hotspot of $\mathrm{AD}$ research is to study the pathogenesis of $\mathrm{AD}$ and seek treatment from the etiology ${ }^{[12]}$.

\section{Pathogenesis of AD:}

The main pathological changes of AD were Neurofibrillary Tangles (NFTs), SPs, neuronal cell loss and gliosis. At present, the etiology of $\mathrm{AD}$ is still unknown, so ideal animal models and treatment methods are lacking. In the past, AD models with the characteristics of nerve fiber tangles, experimental autoimmune dementia and central cholinergic nervous system destruction were mostly used to construct AD models, but the facts showed that these models could not fully reflect the pathological characteristics of AD. There are many studies on the pathogenesis of $A D$ and most of them believe that the toxic effects of $A \beta$ can be used to explain the pathogenesis of $\mathrm{AD}$. The toxic effect of $A \beta$ is the decreased ability to clear the phagocytic virus in $A \beta$ timely and effective manner, resulting in an immune response. $\mathrm{A} \beta$ can cause $\mathrm{Ca}^{2+}$ overload and oxidative stress, a series of physical and chemical processes that further lead to the release of damaging enzymes in the brain, through the accumulation of $A \beta$ in the brain plaque foreign bodies. When the body's own immune system is destroyed, glial cells activate in response, producing more multifactor division, including inflammatory factors. Activated inflammatory factors further accelerate the metabolism of amyloid precursor protein to $A \beta$, such circulatory events that aggravate the inflammatory response. This proves that inflammatory response plays a non-negligible role in the pathogenesis of $\mathrm{AD}$, so it is necessary to effectively control the inflammatory response to delay the accelerated progression of AD.

There are many different theories about the pathogenesis of $\mathrm{AD}$, but the accepted pathogenesis mainly includes inflammatory mechanism, $A \beta$ neurotoxicity hypothesis, free radical damage theory and oxidative stress theory. 
Inflammatory mechanism: Inflammation is the distribution of cells in response to stimuli such as injury or infection. In the rat model of AD, glial cells would produce a large number of SPs in the rat brain tissue and a large number of inflammatory factors would accumulate and activate nearby. Moreover, inflammatory factors would also trigger related brain symptoms of inflammation and bring untold suffering to $\mathrm{AD}$ patients. Main cytokines are involved in the regulation of the inflammatory response in the $\mathrm{AD}$ Interleukin (IL)-6, IL-8, Tumor Necrosis Factor alpha (TNF- $\alpha$ ), Nuclear Factor kappa B (NF- $\kappa B$ ), etc., most of these occurring in the process of inflammation inflammatory factor, generated by the brain cells or participate in brain tissue in patients with $\mathrm{AD}$ can be found in the factor of the existence of these viruses and plays an important role in the process of $A D$ pathogenic, in the process of mediating nerve inflammation increase or reduce the patient's condition.

A neurotoxicity hypothesis: Age spots which produce insoluble plaques in the brain organization, its main composition is extracellular beta-amyloid and this protein in brain tissue local large precipitation accumulated concave plaques; one of the pathogenesis of $\mathrm{AD}$ is associated with age spots generated. $\mathrm{A} \beta$ after precipitation, accumulated strong neurotoxic effect, excessive accumulation in the brain tissue, may induce SPs, NFTs and inflammatory reaction, eventually lead to neuronal degeneration, apoptosis and thus trigger AD. $A \beta$ of physiological functions including kinase activation, the formation of ion channels, cholesterol transport regulation, to a certain extent. On the play to the role of the antioxidant stress, the process of neuron apoptosis must go through three stages, which are shown in the following three aspects: First of all, A $\beta$ has cytotoxic effect, through the interaction between toxic factors can cause neuronal damage speed, but after brain damage matrix, a vicious cycle, accelerating the formation of the AD. Second, increasing a beta gathered will attack the body's immune system, so as to cause local inflammation of the lesions, in fall under the catalysis of nerve cells, resulting in memory and cognitive decline and makes the neuron necrosis, the pathological changes. Finally, $A \beta$ protein's ability to remove germs subsided, the virus will attack to the body of mitochondria, mitochondrial damage and it further leads to the release of destructive enzymes in brain tissue and initiates the process of neuronal apoptosis.

Free radical damage theory: The production and clearance of Reactive Nitrogen Free Radicals (RNS) and Reactive Oxygen Species (ROS) are unbalanced. Oxidative stress occurs when more ROS are produced. The death of brain cells and decline of brain function are caused by the high frequency of free radicals transport in the brain, especially the damage of reactive oxygen radicals, thus it is inferred that the pathogenesis of AD is caused by the high frequency output of reactive oxygen radicals. The production of ROS is mainly through its oxidation characteristics between cells and tissues. The substances in brain cells that can be oxidized include cell membrane, cytoplasm and nucleus. The death of brain cells and the decline of brain function are caused by the oxidation of these substances. During the study of $\mathrm{AD}$, the cause of oxidative stress may lead to a large number of nerve cell death. The reason for the rapid development of $\mathrm{AD}$ is that oxidative stress affects the normal material operation and energy metabolism imbalance in the brain, which damages the cerebral vascular endothelium and leads to insufficient cerebral blood supply. Therefore, the aggravation and development of $\mathrm{AD}$ may be related to the participation of ROS free radicals.

In addition, the study also found that hyperlipidemia, as one of the high risk factors for $\mathrm{AD}$, also plays a crucial role in the onset of AD cholesterol. In addition, further studies showed a positive correlation between the incidence of $\mathrm{AD}$ and serum total cholesterol. Therefore, a low diet may significantly reduce the risk of $\mathrm{AD}$. In recent years, the multi-factor pathogenesis of $\mathrm{AD}$ patients is a composite model obtained by comprehensive modeling. Therefore, this study comprehensively adopted the method of compound modeling to intra-abdominal injection of Zuogui pill by D-galactose and the results obtained by this method are closer to the actual level of AD patients.

\section{Heat Shock Protein 70 (HSP70):}

HSP is a molecular chaperone composed of several highly conserved protein family members. The expression level of HSP70 is closely related to the process of neuron cell death in senile dementia. Neuronal cell death is the main pathway of nerve cell loss and is regulated by nuclear transcription factor NF$\mathrm{kB}$ and amino protease family genes. Therefore, the treatment of senile dementia with TCM has gradually gained the attention of relevant researchers. In the view of TCM, the main cause of senile dementia is insufficiency of kidney essence; while Zuogui pill is a classic prescription for tonifying kidney essence, which conforms to the pathogenesis characteristics of $\mathrm{AD}$. Therefore, medical workers often apply Zuogui pill in 
the treatment of this disease in clinical practice. At the same time, the main ingredients of Zuogui pill include antler gum, Achyranthes bidentata, cooked Rehmannia glutinosa, dodder seed and other beneficial ingredients, which have significant effects in nourishing Yin and nourishing kidney and filling lean pulp, and are very suitable for the treatment of this disease.

\section{AD rat model experiment:}

Selection of experimental animals: For the experimental part of this study, 60 Wistar experimental rats, aged $10 \mathrm{mo}$ and weighing $250 \pm 30 \mathrm{~g}$, were selected from the Animal Experimental Research Center of our province.

Grouping of experimental animals: After $1 \mathrm{w}$ of adaptive feeding, the rats were divided into groups, during which they were free to drink water and eat and drink except for the necessary preparation for the experiment. A total of 12 rats died during feeding and modeling and 48 rats were eventually added to the group. The rats were divided into model group (7), anticerebral failure control group (7), Haboin control group (7), Zuogui pill low-dose group (10), Zuogui pill highdose group (10) and sham operation group (7).

Rat model was established: At the end of adaptive feeding, permanent ligation bands were used to tighten the common arteries of the rats to create a Chronic Cerebral Insufficiency (CBI) model of AD rats. Except for the blank control group, rats in other groups were intraperitoneally injected with $1 \%$ ratio of $32 \mathrm{mg} / \mathrm{kg} / \mathrm{d}$ galactose, while rats in the blank control group were only injected with the same dose of normal saline. Under anesthesia with $3 \%$ sodium phenobarbital, rat heads were disinfected. The skin was cut and the hippocampus was located in the midline of the cranial apex. The skull was drilled and diluted with normal saline to $5 \mu \mathrm{g} / \mu \mathrm{l}$ $\mathrm{A} \beta$. After completing the above procedures, human hippocampi were injected with micro injection within a short time, the surgical site was disinfected, the muscle and skin tissues of the rats were sutured with double ligation with silk thread and the rats were sent to an environment with ventilation facilities for observation. During this period, the control group was still injected with an equal dose of saline and after 4 weeks, the rats were given behavioral tests.

\section{AD rats were screened by water maze test:}

$4 \mathrm{w}$ after the operation, $\mathrm{AD}$ rats were screened by the water maze test and the average evasive performance of the control rats was used as the reference value. The specific implementation steps of the water maze test method are as follows: First, inject water into the tank of the water maze. The water depth can be determined according to the depth of the tank, but it should not be lower than $30 \mathrm{~cm}$. The water temperature should be kept at $25 \pm 2^{\circ}$. Second, after the completion of the test environment, rats swimming in the water maze, make the rat maze familiar environment and to set up good glass platform connected to the sink, sink over through the monitor and the computer connection, when the rats' training space beyond set area, computer become automatic start moving trajectory of the tracer to catch rats. Thirdly, according to the activity track and space range predicted by the system, the movement path of all rats in the tank was calculated automatically. According to the time estimated by the system (incubation period), the movement path was observed continuously for $5 \mathrm{~d}$ and used as the index of learning gene ability. On $6^{\text {th }} d$, the tracking system was withdrawn, but the response values of the spatial exploration ability of the rats were still recorded. Fourthly, the average value of the incubation period of the experimental rats in the platform was calculated and compared with the reference value. The difference between the reference value and the actual measured value was processed to obtain the ratio of the incubation period of the rats hiding the platform to escape. If the value was greater than $18 \%$, the rats were identified as AD rats.

\section{Specimen collection:}

At the end of the water maze test after the last gavage, the rats in each group were selected and the rats with the median score were put to death. The execution process must comply with the code of animal experiment civilization. Executed within $3 \mathrm{~min}$ after take out the rats' brain tissue, according to the proportion of $1: 9$ join physiological saline, made from manual homogenate is $100 \mathrm{~g} / 1$ homogenate, the centrifugal at a speed of $3000 \mathrm{r} / \mathrm{min}$, take the supernatant stored at $4^{\circ}$ to avoid light preservation, on the other side of the brain tissue specimens under $2.5 \%$ dose dialdehyde in fixed, for electron microscopy to observe the ultrastructure.

\section{Transmission Electron Microscope (TEM) Observation:}

Behavior after the test, the rats in all groups randomly selected 30 rats (5) in each group, with $10 \%$ chloral hydrate anesthesia in rats, with $2.5 \%$ glutaraldehyde perfusion fixed phosphate buffer, quickly put to death and brain tissue of rats are extracted, isolated hippocampal tissue, will be isolated from the hippocampus to clean 
phosphate buffer, ultra-thin slicing machine slices after polymerization, observed by TEM to observe and record the value.

\section{Observation indexes:}

The main observation indicators included the number of nuclear transcription factor NF- $\mathrm{NB}$, the number of living neurons, the apoptosis of neurons and the expression of HSP70 in each group. These indicators were analyzed mainly through the determination of brain Acetylcholinesterase (AchE), the ultrastructure of hippocampus and the semi-quantitative analysis results of HSP70 kit.

\section{Statistical treatment:}

Statistical Package for the Social Sciences (SPSS) 23.0 data analysis software was used for data statistics and analysis. The results of data analysis were expressed as $(\mathrm{x} \pm \mathrm{s})$ and the comparison of measured values between groups was conducted in the form of one-way analysis of variance. The comparison of measured values between the two groups was performed by independent sample test and $p<0.05$ was considered statistically significant.

\section{RESULTS AND DISCUSSION}

The results of the average incubation period and the number of times the rats crossed the original platform were compared in Table 1 and fig. 1. As can be seen from the experimental results in the figure, the escape incubation period of the model group rats on the $5^{\text {th }} \mathrm{d}$ and the last $3 \mathrm{~d}$ increased significantly and the number of crossing the original platform also increased significantly. Compared with the control group, these differences were statistically significant $(p<0.05)$. After Zuogui pill treatment, the number of rats crossing the original platform increased significantly in the model group, with statistically significant difference $(\mathrm{p}<0.05)$.
The expression of HSP70 in brain tissue and the detection results of apoptotic neurons are shown in Table 2 and fig. 2 . As can be seen from the experimental results in the figure, no HSP70 protein expression was observed in the sham group and more HSP expression could be found in the cerebral ischemia reperfusion for 24, 48 and $72 \mathrm{~h}$, with the extension of the perfusion time. The expression of HSP70 is gradually weakened. The model group had more HSP expression at each time point and the Optical Density (OD) value of average optical density was higher than that of the corresponding control group, indicating that Zuogui pill could increase the expression of HSP70 in the hippocampus Cornu Ammonis 1 (CA1) region.

The effects of Zuogui pill on the expression of AchE and gene messenger Ribonucleic Acid (mRNA) in rat brain homogenate are shown in Table 3 and fig. 3 . The results showed that AchE activity was abnormally increased in rats after modeling and the difference with blank group was statistically significant $(\mathrm{p}<0.01)$. All kinds of drugs in the model group could significantly inhibit AchE activity in brain tissue, among which Haberine inhibited AchE activity most obviously $(p<0.01)$. The mRNA levels of HSP70 gene in the hippocampus of rats were significantly lower than those in the blank control group. Compared with the model group, the high dose group was slightly higher than the low dose group and the anti-cerebral failure group was slightly higher than the Haberman control group.

The results of learning and memory ability test in $\mathrm{AD}$ rats under the action of Zuogui pill are shown in Table 4 and fig. 4 . As can be seen from the chart, after $14 \mathrm{~d}$ of administration, the learning ability of the model group in the low-dose Zuogui pill group and the highdose Zuogui pill group was significantly improved compared with the photogramme group.

TABLE 1: COMPARISON RESULTS OF AVERAGE INCUBATION PERIOD AND TIMES OF CROSSING THE ORIGINAL PLATFORM IN EACH GROUP ( $\bar{x} \pm s)$

\begin{tabular}{lcccc}
\hline Group & $\mathrm{n}$ & $\begin{array}{c}\mathbf{5} \mathrm{d} \text { escape incubation } \\
\text { period }\end{array}$ & $\begin{array}{c}\text { After 3 d escape } \\
\text { incubation period }\end{array}$ & $\begin{array}{c}\text { Number of platform } \\
\text { locations crossed }\end{array}$ \\
\hline $\begin{array}{l}\text { Model group } \\
\text { (Group A) }\end{array}$ & 7 & $25.33 \pm 2.06$ & $19.75 \pm 3.22$ & $13.41 \pm 1.80$ \\
$\begin{array}{l}\text { Anti-cerebral failure control group } \\
\text { (Group B) }\end{array}$ & 7 & $29.73 \pm 2.16$ & $23.39 \pm 2.53$ & $11.83 \pm 1.16$ \\
$\begin{array}{l}\text { The Haberman control group } \\
\text { (Group C) }\end{array}$ & 7 & $69.43 \pm 4.05$ & $58.08 \pm 3.41$ & $4.68 \pm 0.66$ \\
$\begin{array}{l}\text { Zuogui pill low dose group } \\
\text { (Group D) }\end{array}$ & 10 & $40.06 \pm 3.95$ & $32.02 \pm 2.31$ & $8.85 \pm 0.25$ \\
$\begin{array}{l}\text { Zuogui pill high dose group } \\
\text { (Group E) }\end{array}$ & 10 & $63.98 \pm 5.97$ & $57.72 \pm 1.41$ & $4.23 \pm 0.63$ \\
$\begin{array}{l}\text { Sham operation group } \\
\text { (Group F) }\end{array}$ & 7 & $36.77 \pm 2.93$ & $30.37 \pm 1.42$ & $13.87 \pm 1.22$ \\
\hline
\end{tabular}


www.ijpsonline.com

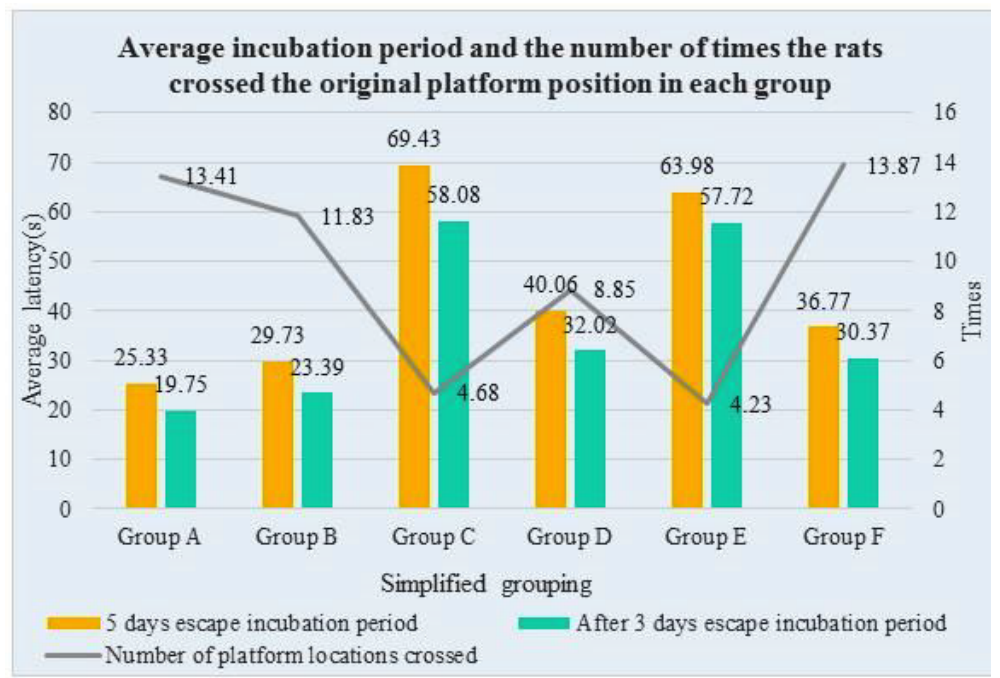

Fig. 1: Comparison results of average incubation period and times of crossing the original platform in each group

TABLE 2: EXPRESSION OF HSP70 IN BRAIN TISSUE AND DETECTION RESULTS OF APOPTOTIC NEURONS (OD VALUE, $\bar{x} \pm s)$

\begin{tabular}{lcccc}
\hline Group & $\mathbf{n}$ & $\mathbf{2 4} \mathrm{h}$ & $\mathbf{4 8} \mathrm{h}$ & $\mathbf{7 2 ~ h}$ \\
\hline Model group (Group A) & 7 & $0.37 \pm 0.03$ & $0.26 \pm 0.03$ & $0.25 \pm 0.02$ \\
Anti-cerebral failure control group (Group B) & 7 & $0.36 \pm 0.04$ & $0.32 \pm 0.03$ & $0.27 \pm 0.03$ \\
The Haberman control group (Group C) & 7 & $0.32 \pm 0.03$ & $0.31 \pm 0.03$ & $0.29 \pm 0.02$ \\
Zuogui pill low dose group (Group D) & 10 & $0.35 \pm 0.02$ & $0.32 \pm 0.02$ & $0.3 \pm 0.02$ \\
Zuogui pill high dose group (Group E) & 10 & $0.36 \pm 0.02$ & $0.33 \pm 0.03$ & $0.31 \pm 0.03$ \\
Sham operation group (Group F) & 7 & 0 & 0 & 0 \\
\hline
\end{tabular}

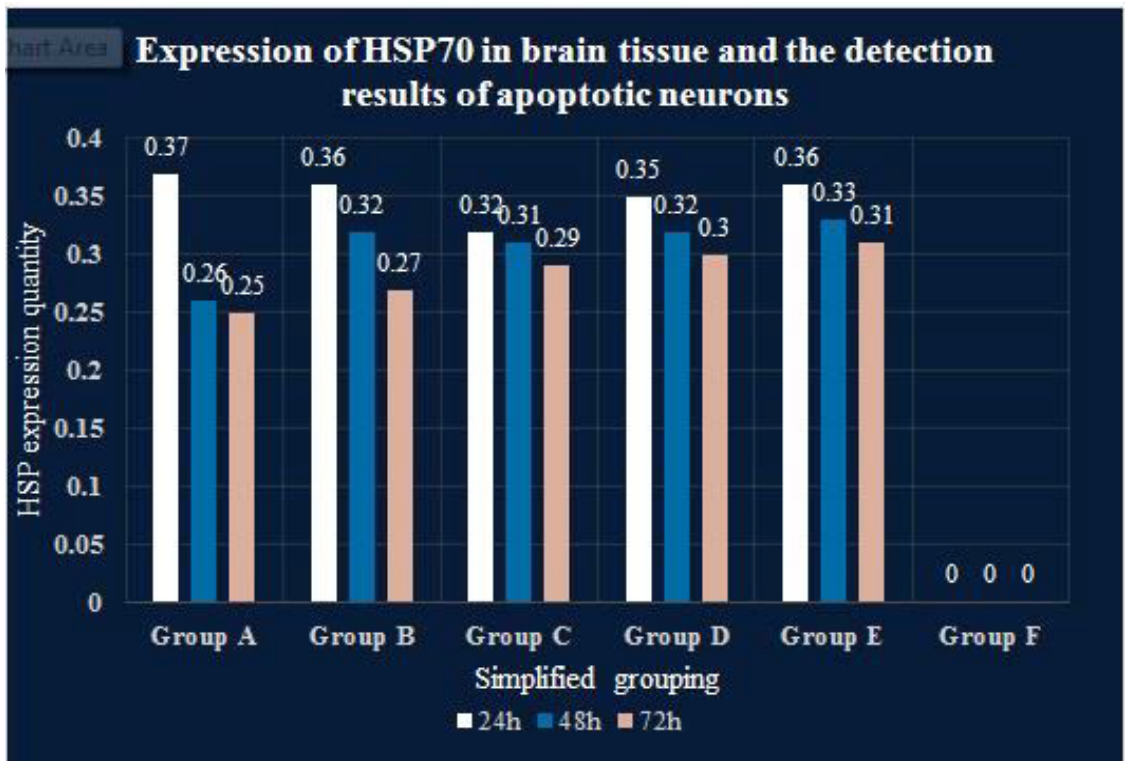

Fig. 2: Expression of HSP70 in brain tissue and detection results of apoptotic neurons

TABLE 3: EFFECT OF ZUOGUI PILL ON AchE AND EXPRESSION OF GENE mRNA IN RAT BRAIN HOMOGENATE ( $\overline{\mathbf{x}} \pm \mathbf{s})$

\begin{tabular}{lccc}
\hline Group & $\mathbf{n}$ & AchE (U/mg) & mRNA \\
\hline Model group (Group A) & 7 & $0.87 \pm 0.21$ & $0.416 \pm 0.058$ \\
Anti-cerebral failure control group (Group B) & 7 & $0.69 \pm 0.22$ & $0.782 \pm 0.837$ \\
The Haberman control group (Group C) & 7 & $0.66 \pm 0.16$ & $0.683 \pm 0.072$ \\
Zuogui pill low dose group (Group D) & 10 & $0.68 \pm 0.14$ & $1.053 \pm 0.104$ \\
Zuogui pill high dose group (Group E) & 10 & $0.7 \pm 0.08$ & $0.936 \pm 0.086$ \\
Sham operation group (Group F) & 7 & $0.56 \pm 0.15$ & $1.537 \pm 0.153$ \\
\hline
\end{tabular}


Effects of Zuogui Pill on AchE and mRNA expression

in rat brain homogenate

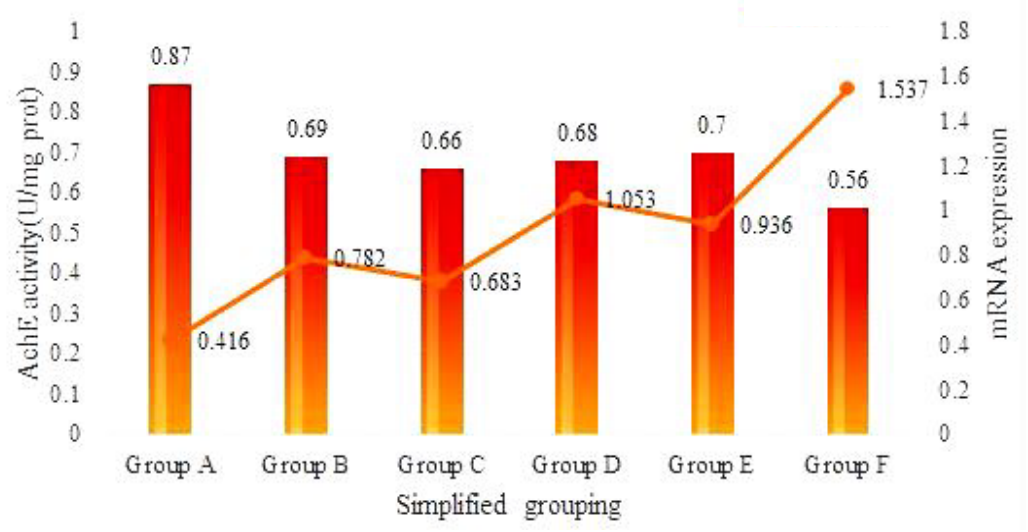

Fig. 3: Effects of Zuogui pill on AchE and mRNA expression of gene in rat brain homogenate; $-\operatorname{AchE}(\mathrm{U} / \mathrm{mg}$ prot $),-$ mRNA

TABLE 4: RESULTS OF LEARNING AND MEMORY ABILITY TEST IN AD RATS ( $\overline{\mathbf{x}} \pm \mathbf{s})$

\begin{tabular}{lcccc}
\hline Group & $\mathbf{n}$ & $\begin{array}{c}\text { Dose } \\
(\mathbf{g} / \mathrm{kg})\end{array}$ & EL (s) & $\begin{array}{c}\text { Total distance } \\
(\mathbf{c m})\end{array}$ \\
\hline Model group (Group A) & 7 & - & $9.35 \pm 1.35$ & $106.26 \pm 21.63$ \\
Anti-cerebral failure control group (Group B) & 7 & - & $43.15 \pm 15.35$ & $462.26 \pm 20.46$ \\
The Haberman control group (Group C) & 7 & 0.083 & $8.35 \pm 2.72$ & $108.35 \pm 17.35$ \\
Zuogui pill low dose group (Group D) & 10 & 0.24 & $29.46 \pm 2.46$ & $305.25 \pm 12.42$ \\
Zuogui pill high dose group (Group E) & 10 & 0.46 & $10.46 \pm 2.62$ & $116.35 \pm 21.64$ \\
Sham operation group (Group F) & 7 & 0.83 & $9.36 \pm 1.73$ & $108.32 \pm 16.32$ \\
\hline
\end{tabular}

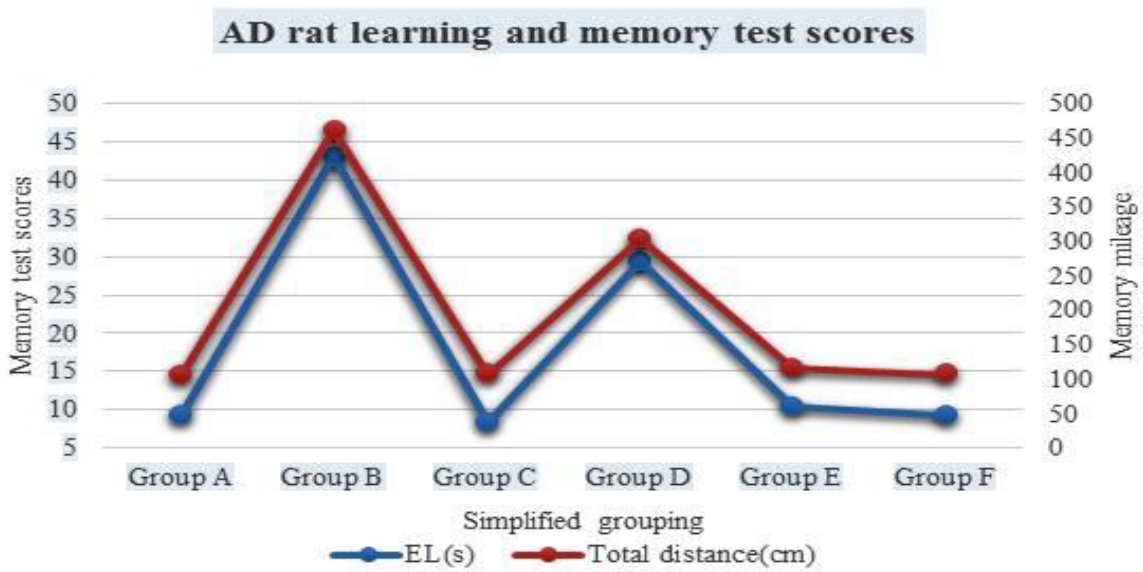

Fig. 4: AD rats' learning and memory ability test scores

The successful establishment of animal model is the basis of objective research results obtained in this study, so the preparation of rat senile dementia model meeting experimental requirements is also the focus of discussion. Neuron loss is the main pathologic change in $\mathrm{AD}$, manifested as memory loss, skin plaques, personality trait changes and cognitive function changes. These pathological features must be simulated in the modeling process. In the study, the authors injected rats with different doses of D-galactose and ensured that the concentration of D-galactose in the body was elevated enough to be reduced to galactose by aldose reductase. This synthetic substance is difficult to be converted into superoxide anions by cell metabolism and abnormal gene expression will lead to decreased cell growth and reproduction ability and then induce the external expression of $\mathrm{AD}$ to be consistent with the ultra structural changes of hippocampus in the brain. Therefore, rats can be used as an animal model to simulate senile dementia. In the process of studying the pathogenesis of $\mathrm{AD}$, it has been found that the main cause of $A D$ is the toxic effect of $A \beta$. After $A \beta$ aggregation effect occurs in brain tissue, neurotoxicity is further developed and pathological symptoms are 
induced. Part of the pathological changes of $\mathrm{AD}$ were simulated by injecting $A \beta_{1-42}$ into the rat brain tissue, and the learning and memory ability of the rats was significantly decreased, and the pathological changes of SPs and NFTs appeared in the rat brain.

For the brain, learning and cognitive acquisition is a multi-stage dynamic process. Learning is a process in which the nervous system, stimulated by the external environment, has a series of effects on itself. Memory function is the process of storing and processing information in the brain. The hippocampus plays an important role in the structure of brain tissue, especially the hippocampus CAI area, which is directly related to spatial discrimination and the ability of learning and memory. When nerve injury occurs, the hippocampus CAI region is the most sensitive and its pathological features are also the most prominent, mainly due to cognitive and memory disorders. Among many test methods to evaluate the learning and memory of rats, Morris water maze experiment is widely used due to its excellent test fairness and this study also adopts this method to evaluate the learning and memory ability of rats.

Deposition of $A \beta$ in the brain tissue of patients with $\mathrm{AD}$ consists of a number of proteins and $\mathrm{A} \beta$, the main components of which include the active substance AchE in and around the plaque increase. In vitro experiments on rat models showed that the complex of AchE and $A \beta$ was more cytotoxic than that of $A \beta$ filament alone. In addition, AchE activates $A \beta$ peptide to assemble into $A \beta$ filament, which can form a complex with AchE of tetramer and has specific protease action. When the molecular distribution of AchE changes, it will affect the generation of $A \beta$. Thus, the enhanced activity of $A \beta$ and surrounding $A c h E$ is $A \beta$ vicious cycle of events leading to pathological changes in AD. Therefore, the abnormal representation of AchE is another important factor causing AD.

In this experiment, the appearance characteristics, brain free radicals and learning ability indexes of the model group rats were observed and it was found that there are many similarities between the natural reaction of senile dementia and human functional aging. And left to pill to significantly improve the effects of the above symptoms, it by reducing brain free radical damage, improve bone metabolism and bone dynamic enhancement, caused by dementia can also improve the ability of learning and memory impairment, prompt out pill has certain anti-aging effect not only, also can tends to senile osteoporosis and physical decline there was a delay effect. In this study, after injecting $A \beta_{1-42}$ into the hippocampal region of rats, the avoidance latency of rats was significantly enhanced and the number of times crossing the original platform was sharply decreased, suggesting that the learning and memory ability of rats was decreased, which was consistent with the results of the modeling hypothesis, indicating that the modeling was successful.

TCM such as Zuogui pill can improve the immune system, regulate endocrine and neurotransmitter oxidation to increase the content of RNA in the brain of patients with $\mathrm{AD}$, thus affecting the expression rules of genes and achieving the purpose of preventing and treating AD. Zuogui pill as a TCM has a significant advantage; it has no obvious toxic side effects. In the experiment of this paper, AchE activity in rat brain tissue was significantly inhibited after drug treatment with Zuogui pill and HSP70 expression was upregulated and apoptosis was inhibited. The results of ultrastructure observation also showed that TCM treatment was more beneficial to the protection of the hippocampus. Therefore, it is sufficient to confirm that Zuogui pill can up-regulate the expression of HSP70 by inhibiting the activity of AchE in the brain tissue of model rats, so as to realize the purpose of inhibiting the apoptosis of neuron cells.

\section{Funding:}

The work was supported by Beijing Hospitals Authority Youth Progranme QMS20211005; and Beijing Association for Science and Technology Young Talent Lifting Proiect.

\section{Conflict of interests:}

The authors declared no conflicts of interest.

\section{REFERENCES}

1. Yan N, Liu Y, Liu S, Cao S, Wang F, Wang Z, et al. Fluorideinduced neuron apoptosis and expressions of inflammatory factors by activating microglia in rat brain. Mol Neurobiol 2016;53(7):4449-60.

2. Kang WH, Cao W, Graudejus O, Patel TP, Wagner S, Meaney $\mathrm{DF}$, et al. Alterations in hippocampal network activity after in vitro traumatic brain injury. J Neurotrauma 2015;32(13):10119.

3. Tian Z, Dong C, Fujita A, Fujita Y, Hashimoto K. Expression of heat shock protein HSP-70 in the retrosplenial cortex of rat brain after administration of (R, S)-ketamine and (S)ketamine, but not (R)-ketamine. Pharmacol Biochem Behav 2018;172:17-21.

4. Wang D, Ripley-Gonzalez JW, Hu Y. Aerobic physical training protects the rat brain against exercise-heat related oxidative damage through the increased expression of HSP70. Neurophysiology 2019;51(2):66-71. 
5. Nam YJ, Lee DH, Shin YK, Sohn DS, Lee CS. Flavanonol taxifolin attenuates proteasome inhibition-induced apoptosis in differentiated PC12 cells by suppressing cell death process. Neurochem Res 2015;40(3):480-91.

6. Boksa P, Zhang Y, Nouel D, Wong A, Wong TP. Early development of Parvalbumin-, somatostatin- and cholecystokinin-expressing neurons in rat brain following prenatal immune activation and maternal Iron deficiency. Dev Neurosci 2016;38(5):342-53.

7. Krityakiarana W, Zhao PM, Nguyen K, Gomez-Pinilla F, Kotchabhakdi N, de Vellis J, et al. Proof-of concept that an acute trophic factors intervention after spinal cord injury provides an adequate niche for neuroprotection, recruitment of nestin-expressing progenitors and regeneration. Neurochem Res 2016;41(1):431-49.

8. Joseph-Bravo P, Jaimes-Hoy L, Charli JL. Regulation of TRH neurons and energy homeostasis-related signals under stress. J Endocrinol 2015;224(3):R139-59.

9. Kanno N, Fujiwara K, Yoshida S, Kato T, Kato Y. Dynamic changes in the localization of neuronatin-positive cells during neurogenesis in the embryonic rat brain. Cells Tissues Organs 2019;207(3-4):127-37.

10. Halasy K, Szőke B, Janzsó G. Fine structure and synaptology of the nitrergic neurons in medial septum of the rat brain. Acta Biol Hung 2017;68(1):1-3.

11. Thakur P, Nehru B. Inhibition of neuroinflammation and mitochondrial dysfunctions by carbenoxolone in the rotenone model of Parkinson's disease. Mol Neurobiol 2015;51(1):20919.

12. Sogut I, Uysal O, Oglakci A, Yucel F, Kartkaya K, Kanbak G. Prenatal alcohol-induced neuroapoptosis in rat brain cerebral cortex: Protective effect of folic acid and betaine. Childs Nerv Syst 2017;33(3):407-17. 